\title{
Electron spin resonance spectroscopy with femtoliter detection volume $\odot$
}

Cite as: Appl. Phys. Lett. 116, 184002 (2020); https://doi.org/10.1063/5.0004322

Submitted: 11 February 2020 . Accepted: 20 March 2020. Published Online: 04 May 2020

V. Ranjan (D), S. Probst, B. Albanese, T. Schenkel (D), D. Vion, D. Esteve, J. J. L. Morton (D), and P. Bertet (D)

\section{COLLECTIONS}

Paper published as part of the special topic on Hybrid Quantum Devices Note: This paper is part of the Special Issue on Hybrid Quantum Devices.

F This paper was selected as Featured
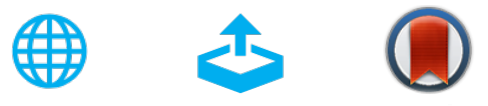

\section{ARTICLES YOU MAY BE INTERESTED IN}

Wavelength transduction from a 3D microwave cavity to telecom using piezoelectric optomechanical crystals

Applied Physics Letters 116, 174005 (2020); https://doi.org/10.1063/5.0002160

\section{A tunable electromagnetic acoustic switch}

Applied Physics Letters 116, 183502 (2020); https://doi.org/10.1063/5.0008532

Realizing isotropic negative thermal expansion covering room temperature by breaking the superstructure of $\mathrm{ZrV}_{2} \mathrm{O}_{7}$

Applied Physics Letters 116, 181902 (2020); https://doi.org/10.1063/1.5143691

\section{Lock-in Amplifiers up to $600 \mathrm{MHz}$}
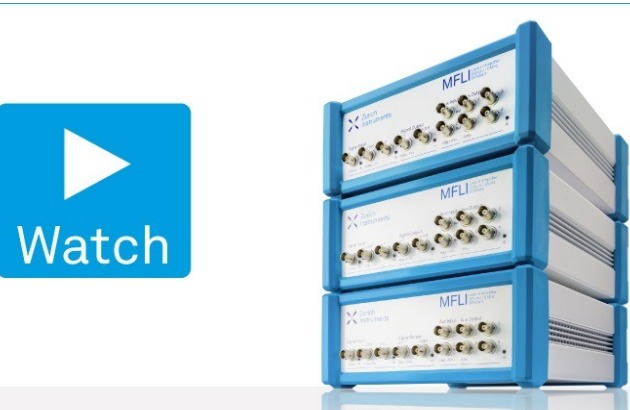


\title{
Electron spin resonance spectroscopy with femtoliter detection volume ?
}

\author{
Cite as: Appl. Phys. Lett. 116, 184002 (2020); doi: 10.1063/5.0004322 \\ Submitted: 11 February 2020 - Accepted: 20 March 2020 . \\ Published Online: 4 May 2020
}

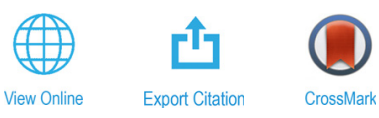

V. Ranjan, ${ }^{1, a)}$ (D) S. Probst, ${ }^{7}$ B. Albanese, ${ }^{7}$ T. Schenkel, ${ }^{2}$ (D) D. Vion, ${ }^{7}$ D. Esteve, ' J. J. L. Morton, ${ }^{3}$ (D) and P. Bertet ${ }^{1, b)}$ (D)

\begin{abstract}
AFFILIATIONS
'Quantronics Group, SPEC, CEA, CNRS, Université Paris-Saclay, CEA Saclay, 91191 Gif-sur-Yvette Cedex, France

${ }^{2}$ Accelerator Technology and Applied Physics Division, Lawrence Berkeley National Laboratory, Berkeley, California 94720, USA

${ }^{3}$ London Centre for Nanotechnology, University College London, London WC1H OAH, United Kingdom
\end{abstract}

Note: This paper is part of the Special Issue on Hybrid Quantum Devices.

a) Author to whom correspondence should be addressed: vishalran@gmail.com

b) patrice.bertet@cea.fr

\begin{abstract}
We report electron spin resonance measurements of donors in silicon at millikelvin temperatures using a superconducting $L C$ planar microresonator and a Josephson parametric amplifier. The resonator includes a nanowire inductor, defining a femtoliter detection volume. Due to strain in the substrate, the donor resonance lines are heavily broadened. Single-spin to photon coupling strengths up to $\sim 3 \mathrm{kHz}$ are observed. The single shot sensitivity is $120 \pm 24$ spins/Hahn echo, corresponding to $\approx 12 \pm 3$ spins $/ \sqrt{\mathrm{Hz}}$ for repeated acquisition.
\end{abstract}

Published under license by AIP Publishing. https://doi.org/10.1063/5.0004322

Electron spin resonance (ESR) spectroscopy is useful for characterizing paramagnetic species and finds applications in a large number of fields. The most widely used detection method is the inductive detection, which relies on the emission of microwave signals by the spins during their Larmor precession into a resonant cavity to which they are magnetically coupled. Conventional inductively detected ESR spectroscopy suffers from a low spin detection sensitivity, which precludes its use for micrometer- or nanoscale samples, ${ }^{1}$ motivating research on alternative detection schemes. ${ }^{2-9}$

Planar micro-resonators ${ }^{10,11}$ and self-resonant microhelices ${ }^{12}$ have been shown to be promising to push inductive detection to higher sensitivity and lower detection volumes, but the microwave confinement that they enable is ultimately limited by Ohmic losses in the metal. This can be overcome by the use of superconducting micro-resonators at low temperatures, ${ }^{13-16}$ for which arbitrarily small detection volumes should, in principle, be achievable while preserving a high resonator quality factor. An additional benefit of using small-mode-volume and high-quality-factor resonators is the enhanced microwave spontaneous emission they cause via the Purcell effect, ${ }^{16-19}$ which enables us to repeat measurements faster and, therefore, impacts favorably the spin detection sensitivity. A recent experiment detected the ESR signal from an ensemble of donors in silicon at millikelvin temperatures coupled to a superconducting resonator with a sub-pL magnetic mode volume, reaching a spin detection sensitivity of $65 \operatorname{spin} / \sqrt{\mathrm{Hz}}$ measured by a Hahn-echo sequence. ${ }^{20}$

Here, we push this effort further with a new resonator geometry incorporating a superconducting nanowire around which the magnetic component of the microwave field is confined, yielding a detection volume as low as $\sim 6 \mathrm{fL}$. Correspondingly, the spin-photon coupling constant reaches values up to $3 \mathrm{kHz}$, an order-of-magnitude enhancement over the state-of-the-art. We estimate a spin detection sensitivity of $12 \pm 3$ spins $/ \sqrt{\mathrm{Hz}}$ for donors in silicon at millikelvin temperatures. As a counterpart, the Rabi frequency is highly inhomogeneous. Also, due to differential thermal contractions between the substrate and the nanowire, the donor resonance is considerably broadened by strain.

An overview of our resonator design ${ }^{16}$ is shown in Fig. 1(b). The resonator is patterned out of a $50 \mathrm{~nm}$-thick superconducting aluminum film. It consists of an interdigitated capacitor $C$ with fingers $10 \mu \mathrm{m}$ wide and separated by the same amount. The capacitor is shorted by a $100 \mathrm{~nm}$-wide, $10 \mu \mathrm{m}$ long wire around which the magnetic component of the microwave field is by far the strongest. Using electromagnetic simulations, ${ }^{21}$ we determine that the resonator total inductance is $L=320 \mathrm{pH}$, with most of it being due to stray inductance in the capacitor. The geometric inductance of the wire alone is estimated to be $10 \mathrm{pH}$, while its kinetic inductance is $30 \mathrm{pH}$. The resulting resonator impedance is $Z_{c} \sim 15 \Omega$. 
(a)

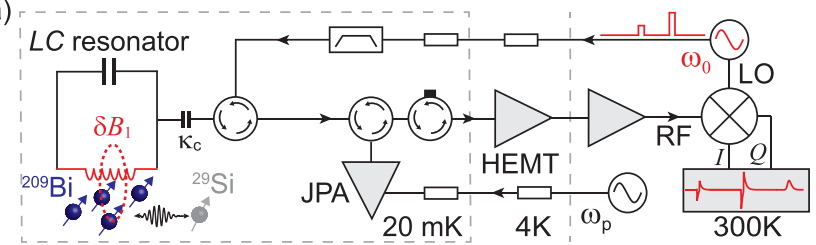

(b)
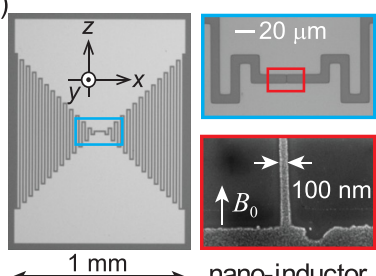

(c)

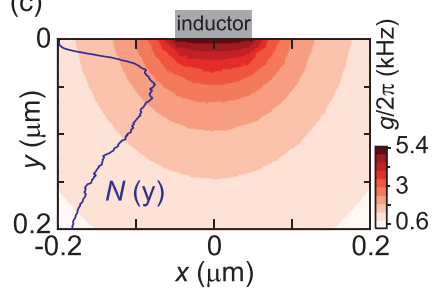

FIG. 1. Quantum limited spectrometer. (a) Schematics of the measurement setup. Square control pulses are applied at resonator frequency $\omega_{0}$. The reflected and emitted signals from spins are amplified by a Josephson parametric amplifier (JPA) in its degenerate mode, i.e., when pumped at $\omega_{p}=2 \omega_{0}$. (b) Optical and scanning electron microscope images of the $L C$ resonator with a nanometric inductor. Light and dark areas form aluminum and the underlying Si substrate, respectively. The magnetic field is applied parallel to the inductor. (c) Calculated spin-resonator coupling strength distribution for the first spin-transition. The implantation profile $N(y)$ with a peak concentration of $8 \times 10^{16} \mathrm{~cm}^{-3}$ is also plotted.

The resonator is patterned on top of a silicon substrate in which bismuth atoms were implanted between 50 and $100 \mathrm{~nm}$ below the surface (see Fig. 1). Bismuth is an electron donor in silicon and can trap the unpaired electron at low temperatures, whose electron spin $S=1 / 2$ provides the ESR signal. ${ }^{22}$ At low magnetic fields $B_{0}$, the ESR-allowed transitions ${ }^{23-26}$ are shown in Fig. 2(a). To increase the donor coherence time, the substrate was enriched in the nuclear-spin-free ${ }^{28} \mathrm{Si}$ isotope, with a nominal residual ${ }^{29} \mathrm{Si}$ relative concentration of $5 \times 10^{-4}$. The sample is cooled at $20 \mathrm{mK}$ and measured using a custom-built ESR spectrometer ${ }^{15,20}$ including a Josephson parametric amplifier ${ }^{27}$ as the first-stage amplification. Note that in this experiment, the maximum microwave input power, $\sim-90 \mathrm{dBm}$, is limited due to the superconducting nanowire that causes a Kerr non-linearity in the resonator response (see the supplementary material for more details). The results from two nearly identical samples (S1 and S2) are reported below; unless mentioned explicitly, the data shown here have been obtained with sample S1.

An important parameter is the spin-photon coupling strength $g_{0}=\gamma_{e}\left\langle 0\left|S_{x}\right| 1\right\rangle \delta B_{1}$, defined as half the Rabi frequency that a spin would undergo in $\delta B_{1}$, the microwave amplitude corresponding to a 1 photon field, with $\gamma_{e} / 2 \pi=28 \mathrm{GHz} / \mathrm{T}$ being the free electron gyromagnetic ratio and $\left\langle 0\left|S_{x}\right| 1\right\rangle$ the spin transition matrix element between the two levels $|0\rangle$ and $|1\rangle$, whose frequency difference is equal to $\omega_{0}$ at the applied $B_{0}$. It is shown in Fig. 1 (c) as a function of the relative position of the donor with respect to the inductor. The magnetic field $\delta B_{1}$ was calculated using an electromagnetic solver, assuming that the ac current corresponding to 1 photon in the resonator $\delta i=\omega_{0} \sqrt{\hbar / 2 Z_{c}}$ flows through the inductor. Close to the nanowire within a depth of $40 \mathrm{~nm}$, $g_{0} / 2 \pi$ reaches values as high as $3-5 \mathrm{kHz}$, one order of magnitude larger than spin-resonator couplings measured so far. ${ }^{20}$ This extreme confinement can be quantified in terms of mode-volume ${ }^{28}$ $\left(\hbar \omega_{0} / 4\right) /\left(\delta B_{1, \max }^{2} / 2 \mu_{0}\right) \approx 12 \mathrm{fL}$, where $\delta B_{1, \max } \sim 0.5 \mu \mathrm{T}$ is calculated
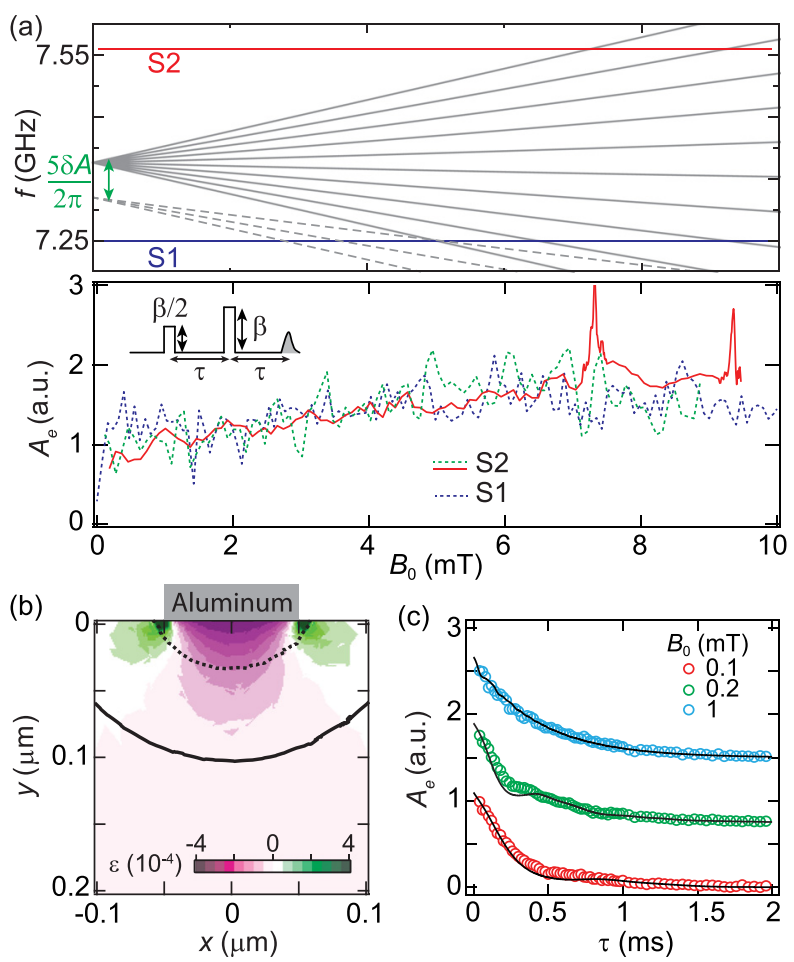

FIG. 2. Spin spectroscopy. (a) Top: ten electron spin transitions of bismuth in the low magnetic field regime. The dashed lines represent new frequencies due to changes in hyperfine constant $\delta A$ by local strain (only three are shown for clarity). Bottom: magnetic field sweep of the echo signal for two devices fabricated on the same substrate (sequence in the inset). Different curves are scaled in amplitude and acquired at different pulse amplitudes $\beta$. For device S2, $\beta=2 \times 10^{5} \mathrm{~s}^{-1 / 2}$ (dashed curve) and $7 \times 10^{5} \mathrm{~s}^{-1 / 2}$ and the pulse duration $d t=0.5 \mu \mathrm{s}$. For device $\mathrm{S} 1, \beta=10^{5} \mathrm{~s}^{-1 / 2}$ and $d t=1 \mu \mathrm{s}$. (b) Hydrostatic component $\epsilon$ of the strain computed using finite element model simulations of COMSOL software. Solid and dashed curves represent the respective contours of probed spins in S2 when assuming perfect $\pi$ pulses for two $\beta$ values. (c) Two pulse spin coherence measurements with S1: echo area $A_{\mathrm{s}}$ as a function of delay $\tau$. Curves have been offset for clarity. Solid curves are simulations.

using electromagnetic simulations. The ESR detection volume is thus $6 \mathrm{fL}$ since spins are located only in the lower half of the substrate. The price to pay, however, is a large spatial inhomogeneity of $g_{0}$. Consequences of a wide distribution of $g_{0}$ values when combined with spin relaxation by the Purcell effect were analyzed in Ref. 29 for typical pulse ESR sequences. Consider a two-pulse-echo sequence, consisting of a first microwave pulse of amplitude $\beta / 2$ and duration $d t$, followed by a waiting time $\tau$ and by a second pulse of amplitude $\beta$ and duration $d t$ [see Fig. 2(a)] with $\beta=\sqrt{P_{\text {in }} / \hbar \omega_{0}}\left(\beta^{2}\right.$ represents the number of incoming photons per second). Because of the $g_{0}$ inhomogeneous distribution, the spin echo signal observed a time $\tau$ after the second pulse receives its dominant contribution from spins with a coupling constant $g_{0}(\beta)=\pi \sqrt{\kappa} /(4 \beta d t)$ because those undergo Rabi angles close to $\pi / 2$ and $\pi$. It is, therefore, possible to probe spins with different coupling constants by changing the amplitude $\beta$ of the detection echo sequence. $^{29}$

A first example of this selectivity is provided by spectrum measurements shown in Fig. 2(a). The integral $A_{\mathrm{e}}$ of a spin-echo is 
displayed as a function of the value of the magnetic field $B_{0}$, applied parallel to the inductor. Two spectra measured in S2 are displayed, for two values of $\beta$ (corresponding to input powers of $-100 \mathrm{dBm}$ and $-86 \mathrm{dBm}$ ). In the high-power spectrum, two narrow peaks (solid line) are observed close to the expected bismuth donor ESR transitions, on top of an approximately constant signal that extends until $B_{0}=0$. In the low-power spectrum, the peaks vanish and only the nearly constant echo signal is observed. Only the low-power curve was measured with resonator S1, and a spectrum similar to the one of sample S2 is observed [see Fig. 2(a)].

These observations suggest that bismuth donors spin closest to the wire (those detected in the low-power measurement) have a very broad spectrum, whereas those far from the wire (detected in the highpower measurement) have a narrower linewidth. This can be qualitatively understood by the effect of mechanical strain on the spin properties of bismuth donors. The hyperfine constant was recently shown ${ }^{30}$ to depend linearly on the hydrostatic strain $\epsilon$, with $(d A / d \epsilon) /$ $2 \pi \sim 29 \mathrm{GHz}$. Aluminum contracts ten times more than silicon upon cooldown from room temperature to $10 \mathrm{mK}$. The calculated strain profile $\epsilon$ in silicon, resulting from the differential thermal contraction of the aluminum inductor patterned on top, is shown in Fig. 2(b). ${ }^{31}$ For spins located in the region close to the inductance (and therefore strongly coupled to the resonator), the standard deviation in the zerofield splitting (equal to $5 A / 2 \pi$ ) is $\sim 100 \mathrm{MHz}$, which is sufficient to account for a complete overlap of neighboring peaks and therefore a nearly flat spectrum. Spins further from the inductor are subjected to much lower strain, leading to better-resolved transitions. Because of the peak overlap, each of the 10 Bismuth transitions contributes to the echo signal [see Fig. 2(a)], which we take into account in the sensitivity analysis below. Strain could be mitigated by the choice of metal with a smaller thermal contraction such as niobium.

To confirm that the echo signals arise from implanted bismuth and not from surface impurities, we get information about the spin environment using hyperfine spectroscopy. The integrated spin-echo amplitude $A_{e}$ is measured as a function of $\tau$ at various $B_{0}$ values [Fig. 2(c)]. For $B_{0}<1 \mathrm{mT}$, slight oscillations are visible on top of an exponential decay with $T_{2}=0.85 \mathrm{~ms}$. They approximately match the expected Electron Spin Echo Envelope Modulation (ESEEM) by a bath of ${ }^{29} \mathrm{Si}$ nuclei with the nominal relative concentration of $5 \times 10^{-4}, 32$ suggesting that spins are in the bulk of the sample.

In the Purcell regime, $T_{1}=\kappa /\left(4 g_{0}^{2}\right)$ at resonance, implying that the spin-photon coupling constant can be deduced from spin relaxation measurements. The corresponding pulse sequence consists of a long saturation pulse, followed after a delay $T$ by a detection-echo whose pulse amplitude $\beta_{0}=6 \times 10^{4} \mathrm{~s}^{-1 / 2}\left(P_{\text {in }} \approx-107 \mathrm{dBm}\right.$, in the resonator linear regime) mostly selects a class of spins with coupling constant $g_{0}\left(\beta_{0}\right)$. The integrated echo is shown in Fig. 3(a) as a function of $T$. An exponential fit yields $T_{1}=2 \pm 0.4 \mathrm{~ms}$, which translates into a coupling constant of $g_{0}\left(\beta_{0}\right) / 2 \pi=2.7 \mathrm{kHz}$. This is the largest spin-photon magnetic coupling measured, confirming the predicted coupling distribution [Fig. 1(c)]. Larger values may be obtained using superconducting flux-qubits. ${ }^{33}$ As discussed in Ref. 29, the measured relaxation time scales as $\beta^{2}$, in good agreement with simulations. This enables us to calibrate $\beta$ in absolute units, which is, otherwise, difficult because of the imperfect knowledge of the total attenuation in the input line. The validity of the calibration is confirmed by a Rabi nutation experiment, measured with a pulse sequence shown in Fig. 3(b).
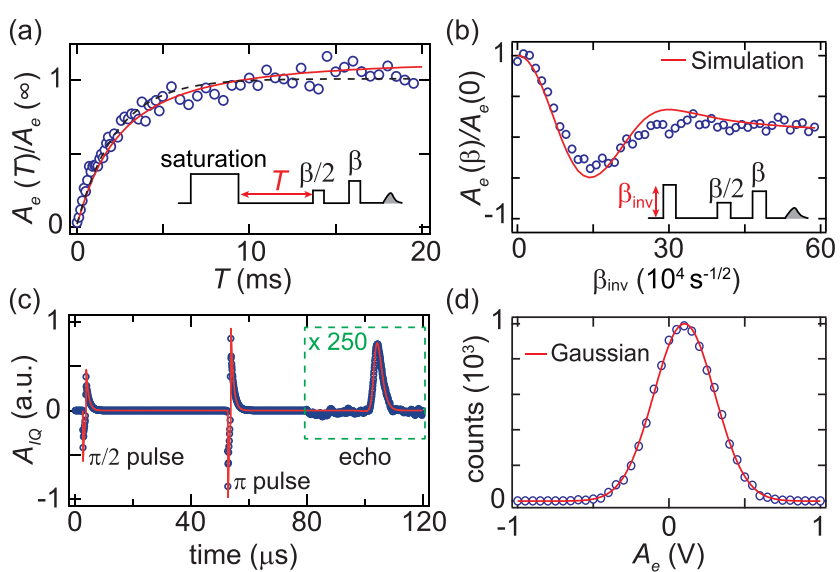

FIG. 3. Spectrometer sensitivity. (a) Spin relaxation time measured using the saturation recovery method. The dashed curve is the best fit to an exponential yielding $T_{1}=2 \pm 0.4 \mathrm{~ms}$. (b) Rabi oscillations performed using three pulse sequence shown in the inset at $\gamma_{\text {rep }}=50 \mathrm{~Hz}$. (c) Measurement of the reflected amplitude $A_{I Q}$ showing a complete Hahn echo sequence. (d) Histograms of echoes at $\gamma_{\text {rep }}=100 \mathrm{~Hz}$ measured with phase cycling. Solid curves in panels (a)-(c) are numerical simulations taking into account all ten ESR transitions.

The simulation agrees quantitatively with the data, without any adjustable parameter.

We then estimate the spectrometer sensitivity following the method explained in Ref. 20. The number of spins $N_{\text {spin }}$ contributing to an echo signal, defined as the total number of spins excited after the initial pulse is first determined: we measure a complete Hahn-echo sequence, including two control pulses of amplitude $\beta_{0} / 2, \beta_{0}$ and duration $1 \mu$ s separated by $50 \mu \mathrm{s}$. The JPA was switched off to avoid its saturation during the application of the control pulses. The reflected amplitude, obtained after $10^{6}$ averages measured with a repetition rate of $100 \mathrm{~Hz}$, is shown in Fig. 3(c). Since the ratio between the echo and control pulse amplitude is uniquely determined by $N_{\text {spin }}$, the latter is obtained by adjusting the simulations to best fit the data [see the solid line in Fig. $3(\mathrm{c})$ ], yielding $N_{\text {spin }} \approx 36 \pm 8$. We note that all ten transitions are equally weighted to account for the overlap of the ESR transition due to strain-induced large spectral broadening. This number is also roughly consistent with the number of bismuth atoms expected in the resonator magnetic mode volume and bandwidth.

The signal-to-noise ratio (SNR) is measured by acquiring $10^{4}$ echo traces (with phase cycling) in the degenerate mode of the JPA at $\gamma_{\text {rep }}=100 \mathrm{~Hz}$. From the histogram shown in Fig. 3(d), we find that the SNR is 0.33 for a single echo trace. Therefore, one could detect $N_{\text {min }} \approx 120 \pm 24$ spins with unity SNR in a single echo sequence. Since the measurements are repeated with a repetition rate of $100 \mathrm{~Hz}$, this translates into a spin detection sensitivity of $12 \pm 3$ spins $/ \sqrt{\mathrm{Hz}}$. Theoretical estimates of the sensitivity ${ }^{15}$ predict that $N_{\min }=\frac{\kappa}{2 P g_{0}} \sqrt{\tilde{n}}$, with $\tilde{n}=1 / 2$ being the noise due to quantum fluctuations of the microwave field and $P \approx 1$, the thermal polarization of the spins at $20 \mathrm{mK}$. For $g_{0} / 2 \pi=2.7 \mathrm{kHz}$, this yields $N_{\min }=50$, in semiquantitative agreement with the measured value. Further improvements in sensitivity would require to further increase $g_{0}$ or the quality factor or to reduce the noise below the quantum limit using squeezed vacuum for instance, as demonstrated recently. ${ }^{34}$ 

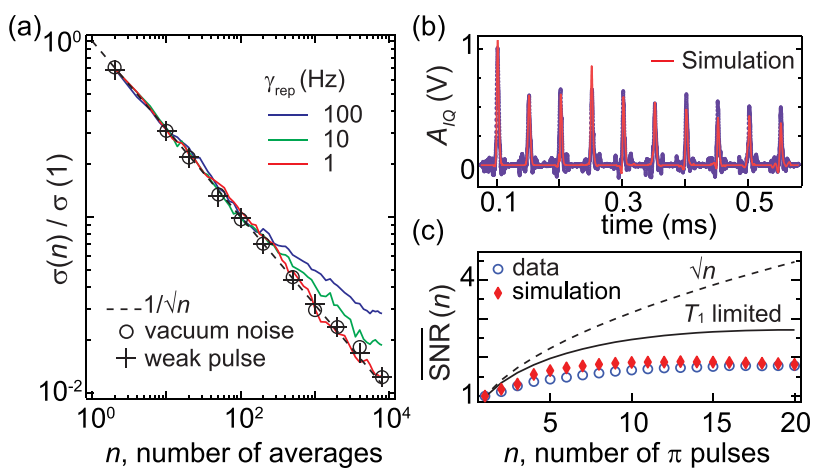

FIG. 4. Correlated noise and CPMG sequences. (a) Measured standard deviation of signals vs number $n$ of averages shown by solid lines and symbols. (b) In symbols, the averaged amplitude of $10^{4}$ repetitions of CPMG traces measured at $\gamma_{\text {rep }}=100 \mathrm{~Hz}$ in the degenerate mode of the JPA. Refocusing pulses are not visible due to phase cycling. (c) Improvement of the echo SNR with the number of refocusing $\pi$ pulses. Simulated SNR (diamond symbols) and calculated curves for cases of no energy relaxation (dashed line) and finite relaxation (solid line), assuming uncorrelated noise between echoes.

Expressing the spin sensitivity in spin $/ \sqrt{\mathrm{Hz}}$ assumes that repeating the same sequence $n$ times and averaging the result increases the SNR by $\sqrt{n}$. We test this assumption by acquiring $10^{7}$ echo sequences, repeated with a rate of $100 \mathrm{~Hz}$, generating a histogram obtained by averaging $n$ consecutive echo integrals, and computing the standard deviation $\sigma(n)$. The result is shown in Fig. 4(a). We observe that, while $\sigma(n)$ indeed scales like $1 / \sqrt{n}$ until $n=200$, it keeps going down for larger values of $n$ but slower than $1 / \sqrt{n}$.

To test whether the deviation of $\sigma(n)$ from $1 / \sqrt{n}$ is due to the setup or to the sample, we mimic the echo acquisition by sending a train of weak coherent pulses with an amplitude that corresponds to an echo and the same repetition rate of $100 \mathrm{~Hz}$ at the resonator frequency $\omega_{0}$. Their standard deviation now follows the $1 / \sqrt{n}$ law until at least $n=10^{4}$, implying that the slower-than $-\sqrt{n}$ echo averaging is not due to setup drift. Note that compared (and contrary) to the analysis performed in Ref. 20, the test pulses were sent at the resonator frequency $\omega_{0}$ so that they would be affected by resonator phase noise, which can, thus, be ruled out as the origin of the slower-than- $\sqrt{n}$ echo signal averaging.

Further insight is obtained by analyzing the spin-echo data differently: instead of averaging $n$ consecutive echo traces, we average them with a separation of 10 or 100 traces (which amounts to effectively changing the repetition rate to $10 \mathrm{~Hz}$ or $1 \mathrm{~Hz}$ ). As seen in Fig. 4(a), the $1 / \sqrt{n}$ law is progressively recovered. A possible interpretation is that the number of spins contributing to the echo slightly fluctuates over a timescale of a few seconds, possibly due to a slow redistribution of the bismuth donor population within the hyperfine states or to ionization/ neutralization dynamics of one or a few donors located close to the metallic electrodes.

One way to increase the spectrometer SNR is to add extra refocusing pulses after the emission of the Hahn echo in order to obtain several echoes per sequence. ${ }^{15,35}$ To this end, we use a Carr-PurcellMeiboom-Gill (CPMG) sequence: $(\pi / 2)_{ \pm x}-\tau-\pi_{y}-\tau-(-$ echo $\left.-\tau / 2-\pi_{y}-\tau / 2-\right)_{n}-e c h o$. The echo train generated by this sequence is shown as symbols in Fig. 4(b) for $\tau=50 \mu$ s (the refocusing pulses are not visible due to phase cycling). The numerical simulation plotted as a solid line describes well the change in the amplitude over time, without any adjustable parameter other than the overall amplitude. Note that the echo amplitude quickly decays after the first pulse, due to the large pulse errors caused by the $B_{1}$ inhomogeneity. In order to quantify the SNR improvement, we measure $10^{4}$ sequences of CPMG echoes (with a repetition rate of $\gamma_{\text {rep }}=100 \mathrm{~Hz}$ ). We then generate histograms obtained by averaging the first $n$ echoes of each sequence and divide the mean by the standard deviation, yielding the SNR as a function of $n$. We find a maximum SNR improvement of $\approx 2$, which corresponds to a spin detection sensitivity of 6 spins $/ \sqrt{\mathrm{Hz}}$. This enhancement is well reproduced by simulations and is not far from the maximum limit $\sim 2.7$ set by the energy relaxation [see Fig. 4(c)].

In summary, we demonstrate a sensitivity of 12 spins $/ \sqrt{\mathrm{Hz}}$ in inductively detected ESR spectroscopy, using a resonator based on a superconducting nanowire with a detection volume of $6 \mathrm{fL}$. The spinresonator coupling reaches values up to $3 \mathrm{kHz}$. An ESEEM signal originating from $\sim 30$ electron spins coupled to residual ${ }^{29} \mathrm{Si}$ nuclear spins was detected. Future work will target further sensitivity enhancement by reducing the nanowire dimensions, as well as possible applications of superconducting ESR spectroscopy to real-world systems, for instance, paramagnetic defects in two-dimensional van der Waals materials.

See the supplementary material for more details on resonator characterization, the bismuth spin system, and the measurement setup.

We acknowledge technical support from P. Sénat and P.-F. Orfila and discussions within the Quantronics group. We thank the European Research Council under the European Community's Seventh Framework Programme (No. FP7/2007-2013) through Grant Agreement Nos. 615767 (CIRQUSS), 279781 (ASCENT), and 630070 (quRAM) of the ANR projects QIPSE and NASNIQ (Contract No. ANR-17-CHIN-0001). T.S. was supported by the U.S. Department of Energy under Contract No. DE-AC02-05CH11231.

The data that support the findings of this study are available from the corresponding author upon reasonable request.

\section{REFERENCES}

${ }^{1}$ A. Schweiger and G. Jeschke, Principles of Pulse Electron Paramagnetic Resonance (Oxford University Press, 2001).

${ }^{2}$ J. Wrachtrup, C. Von Borczyskowski, J. Bernard, M. Orritt, and R. Brown, Nature 363, 244 (1993).

${ }^{3}$ A. Gruber, A. Dräbenstedt, C. Tietz, L. Fleury, J. Wrachtrup, and C. V. Borczyskowski, Science 276, 2012 (1997).

${ }^{4}$ D. Rugar, C. Yannoni, and J. Sidles, Nature 360, 563 (1992).

${ }^{5}$ D. Rugar, R. Budakian, H. Mamin, and B. Chui, Nature 430, 329 (2004).

${ }^{6}$ R. V. Chamberlin, L. A. Moberly, and O. G. Symko, J. Low Temp. Phys. 35, 337 (1979).

${ }^{7}$ Y. Manassen, R. J. Hamers, J. E. Demuth, and A. J. Castellano, Jr., Phys. Rev. Lett. 62, 2531 (1989).

${ }^{8}$ S. Baumann, W. Paul, T. Choi, C. P. Lutz, A. Ardavan, and A. J. Heinrich, Science 350, 417 (2015).

${ }^{9}$ A. Morello, J. J. Pla, F. A. Zwanenburg, K. W. Chan, K. Y. Tan, H. Huebl, M. Mottonen, C. D. Nugroho, C. Yang, J. A. van Donkelaar et al., Nature 467, 687 (2010).

${ }^{10}$ R. Narkowicz, D. Suter, and I. Niemeyer, Rev. Sci. Instrum. 79, 084702 (2008).

${ }^{11}$ Y. Artzi, Y. Twig, and A. Blank, Appl. Phys. Lett. 106, 084104 (2015). 
${ }^{12}$ J. W. Sidabras, J. Duan, M. Winkler, T. Happe, R. Hussein, A. Zouni, D. Suter, A. Schnegg, W. Lubitz, and E. J. Reijerse, Sci. Adv. 5, eaay1394 (2019).

${ }^{13}$ H. Malissa, D. I. Schuster, A. M. Tyryshkin, A. A. Houck, and S. A. Lyon, Rev. Sci. Instrum. 84, 025116 (2013).

${ }^{14}$ A. J. Sigillito, H. Malissa, A. M. Tyryshkin, H. Riemann, N. V. Abrosimov, P. Becker, H.-J. Pohl, M. L. W. Thewalt, K. M. Itoh, J. J. L. Morton, A. A. Houck, D. I. Schuster, and S. A. Lyon, Appl. Phys. Lett. 104, 222407 (2014).

${ }^{15}$ A. Bienfait, J. Pla, Y. Kubo, M. Stern, X. Zhou, C.-C. Lo, C. Weis, T. Schenkel, M. Thewalt, D. Vion, D. Esteve, B. Julsgaard, K. Moelmer, J. Morton, and P. Bertet, Nat. Nanotechnol. 11, 253 (2016).

${ }^{16}$ C. Eichler, A. J. Sigillito, S. A. Lyon, and J. R. Petta, Phys. Rev. Lett. 118, 037701 (2017).

${ }^{17}$ E. M. Purcell, Phys. Rev. 69, 37 (1946).

${ }^{18}$ P. Goy, J. M. Raimond, M. Gross, and S. Haroche, Phys. Rev. Lett. 50, 1903 (1983).

${ }^{19}$ A. Bienfait, J. Pla, Y. Kubo, X. Zhou, M. Stern, C.-C. Lo, C. Weis, T. Schenkel, D. Vion, D. Esteve, J. Morton, and P. Bertet, Nature 531, 74 (2016).

${ }^{20}$ S. Probst, A. Bienfait, P. Campagne-Ibarcq, J. J. Pla, B. Albanese, J. F. D. S. Barbosa, T. Schenkel, D. Vion, D. Esteve, K. Moelmer, J. J. L. Morton, R. Heeres, and P. Bertet, Appl. Phys. Lett. 111, 202604 (2017).

${ }^{21}$ CST Microwave Studios ${ }^{\circledR}$, “CST Microwave Studio," CST Studio Suite, 2008, available at http://www.cst.com.

${ }^{22}$ G. Feher, Phys. Rev. 114, 1219 (1959).

${ }^{23}$ G. W. Morley, M. Warner, A. M. Stoneham, P. T. Greenland, J. van Tol, C. W. Kay, and G. Aeppli, Nat. Mater. 9, 725 (2010).

${ }^{24}$ M. H. Mohammady, G. W. Morley, and T. S. Monteiro, Phys. Rev. Lett. 105, 067602 (2010).

${ }^{25}$ R. E. George, W. Witzel, H. Riemann, N. V. Abrosimov, N. Nötzel, M. L. W. Thewalt, and J. J. L. Morton, Phys. Rev. Lett. 105, 067601 (2010).
${ }^{26}$ G. Wolfowicz, A. M. Tyryshkin, R. E. George, H. Riemann, N. V. Abrosimov, P. Becker, H.-J. Pohl, M. L. W. Thewalt, S. A. Lyon, and J. J. L. Morton, Nat. Nanotechnol, 8, 561 (2013).

${ }^{27}$ X. Zhou, V. Schmitt, P. Bertet, D. Vion, W. Wustmann, V. Shumeiko, and D. Esteve, Phys. Rev. B 89, 214517 (2014).

${ }^{28} \mathrm{~S}$. Haroche and J. M. Raimond, Exploring the Quantum (Oxford University Press, 2006), Sec. 3.1.1.

${ }^{29}$ V. Ranjan, S. Probst, B. Albanese, A. Doll, O. Jacquot, E. Flurin, R. Heeres, D. Vion, D. Esteve, J. J. L. Morton, and P. Bertet, J. Magn. Reson. 310, 106662 (2020).

${ }^{30}$ J. Mansir, P. Conti, Z. Zeng, J. Pla, P. Bertet, M. Swift, C. Van de Walle, M. Thewalt, B. Sklenard, Y. Niquet, and J. Morton, Phys. Rev. Lett. 120, 167701 (2018).

${ }^{31}$ J. Pla, A. Bienfait, G. Pica, J. Mansir, F. Mohiyaddin, Z. Zeng, Y. Niquet, A. Morello, T. Schenkel, J. Morton, and P. Bertet, Phys. Rev. Appl. 9, 044014 (2018).

${ }^{32}$ S. Probst, G. L. Zhang, M. Rancic, V. Ranjan, M. L. Dantec, Z. Zhong, B. Albanese, A. Doll, R. B. Liu, J. J. L. Morton, T. Chanelilere, P. Goldner, D. Vion, D. Esteve, and P. Bertet, arXiv:2001.04854 (2020).

${ }^{33}$ X. Zhu, S. Saito, A. Kemp, K. Kakuyanagi, S-i Karimoto, H. Nakano, W. J. Munro, Y. Tokura, M. S. Everitt, K. Nemoto, M. Kasu, N. Mizuochi, and K. Semba, Nature 478, 221 (2011).

${ }^{34}$ A. Bienfait, P. Campagne-Ibarcq, A. Kiilerich, X. Zhou, S. Probst, J. Pla, T. Schenkel, D. Vion, D. Esteve, J. Morton, K. Moelmer, and P. Bertet, Phys. Rev. X 7, 041011 (2017)

${ }^{35}$ F. Mentink-Vigier, A. Collauto, A. Feintuch, I. Kaminker, V. Tarle, and D. Goldfarb, J. Magn. Reson. 236, 117 (2013).

${ }^{36}$ A. K. Geim and I. V. Grigorieva, Nature 499, 419 (2013). 